\title{
Hardware-in-the-Loop Simulation Using Real-Time Hybrid-Simulator for Dynamic Performance Test of Power Electronics Equipment in Large Power System
}

\author{
Jiyoung Song ${ }^{1,2}$, Kyeon Hur ${ }^{3}{ }^{(D}$, Jeehoon Lee ${ }^{3}$, Hyunjae Lee ${ }^{3}$, Jaegul Lee ${ }^{1,2}$, Solyoung Jung ${ }^{2}$, \\ Jeonghoon Shin ${ }^{2}$ and Heejin Kim ${ }^{3, *}$ \\ 1 School of Electrical Engineering, Korea University, Seoul 02841, Korea; jy.song@kepco.co.kr (J.S.); \\ jaegul.lee@kepco.co.kr (J.L.) \\ 2 Korea Electric Power Corporation (KEPCO), Daejeon 34056, Korea; solyoung.jung@kepco.co.kr (S.J.); \\ jeonghoon.shin@kepco.co.kr (J.S.) \\ 3 School of Electrical and Electronic Engineering, Yonsei University, Seoul 34056, Korea; \\ khur@yonsei.ac.kr (K.H.); jeehun87@yonsei.ac.kr (J.L.); newbee123@yonsei.ac.kr (H.L.) \\ * Correspondence: jimmykim07@yonsei.ac.kr; Tel.: +82-2-2123-8396
}

Received: 22 June 2020; Accepted: 31 July 2020; Published: 1 August 2020

\begin{abstract}
This paper presents the hardware-in-the-loop simulation for dynamic performance test (HILS-DPT) of power electronic equipment replicas using a real-time hybrid simulator (RTHS). The authors developed the procedure of HILS-DPT, and as an actual case example, the results of HILS-DPT of Static VAR Compensator (SVC) replica using RTHS is presented. RTHS is a co-simulation tool that synthesizes real-time simulator (RTS) with transient stability program to perform real-time dynamic simulation of a large power system. As power electronics applications have been increasing, the electric utilities have performed HILS-DPT of the power electronics equipment to validate the performance and investigate interactions. Because inspection tests are limited in their ability to validate its impact on the power system during various contingencies, all power electronics equipment newly installed in the Korean power system should take HILS-DPT using large-scale RTS with replicas since 2018. Although large-scaled RTS offers an accuracy improvement, it requires lots of hardware resources, time, and effort to model and simulate the equipment and power systems. Therefore, the authors performed SVC HILS-DPT using RTHS, and the result of the first practical application of RTHS present feasibility comparing the result of HILS-DPT using large-scale RTS. The authors will discuss the test results and share lessons learned from the industrial experience of HILS-DPT using RTHS.
\end{abstract}

Keywords: real-time hybrid-simulator (RTHS); hybrid simulation; co-simulation; hardware-in-the-loop simulation (HILS); dynamic performance test (DPT); real-time simulator (RTS); testing of replicas

\section{Introduction}

As the power system has become larger and more complicated, the power electronic devices, such as high-voltage direct current (HVDC) and flexible AC transmission systems (FACTS), have been increased to improve stability and controllability and flexibility of the power systems. In the Korean power system, three HVDCs and eleven FACTSs are in operation, four HVDCs are under construction, and two HVDCs and seven FACTSs are under planning [1]. Power electronics applications are located close to each other in the Korean power system, and concerns about interaction with adjacent network dynamics among the equipment have increased [1-8]. The hardware-in-the-loop tests of the power electronics equipment controller are required to demonstrate the equipment performance 
and validate the reliability of the equipment. Therefore, power electronics equipment planned to be newly installed in the Korean power system has had to take a hardware-in-the-loop simulation dynamic performance test (HILS-DPT) of the replica controller using a large-scale real-time simulation (RTS) since 2018 [2]. Herein, we define DPT as a test that validates controller performance using HILS, considering the power system dynamics of other equipment. Figure 1 shows the configuration of DPT. DPT should include the system-wide impact study for multiple power system scenarios with various contingencies. Therefore, DPT requires a large-scale RTS with extensive hardware resources, time, and effort for modeling and simulation. KEPCO has enough RTS hardware resources to accommodate the entire Korean network over a $154 \mathrm{kV}$ transmission system and several HVDC and FACTS without equivalent network and improved the process of simulation case development for efficient modeling and simulation. However, the power system becomes larger and larger, and the system configuration also becomes more and more complicated by applying various power electronics devices, such as HVDC, FACTS, and renewables. The scale of RTS needs to be expanded to analyze the complicated and expanded future power system, and expanding the RTS requires high techniques for modeling and simulation. To overcome the issue, the authors investigated and explored the feasibility of applying to HILS-DPT.

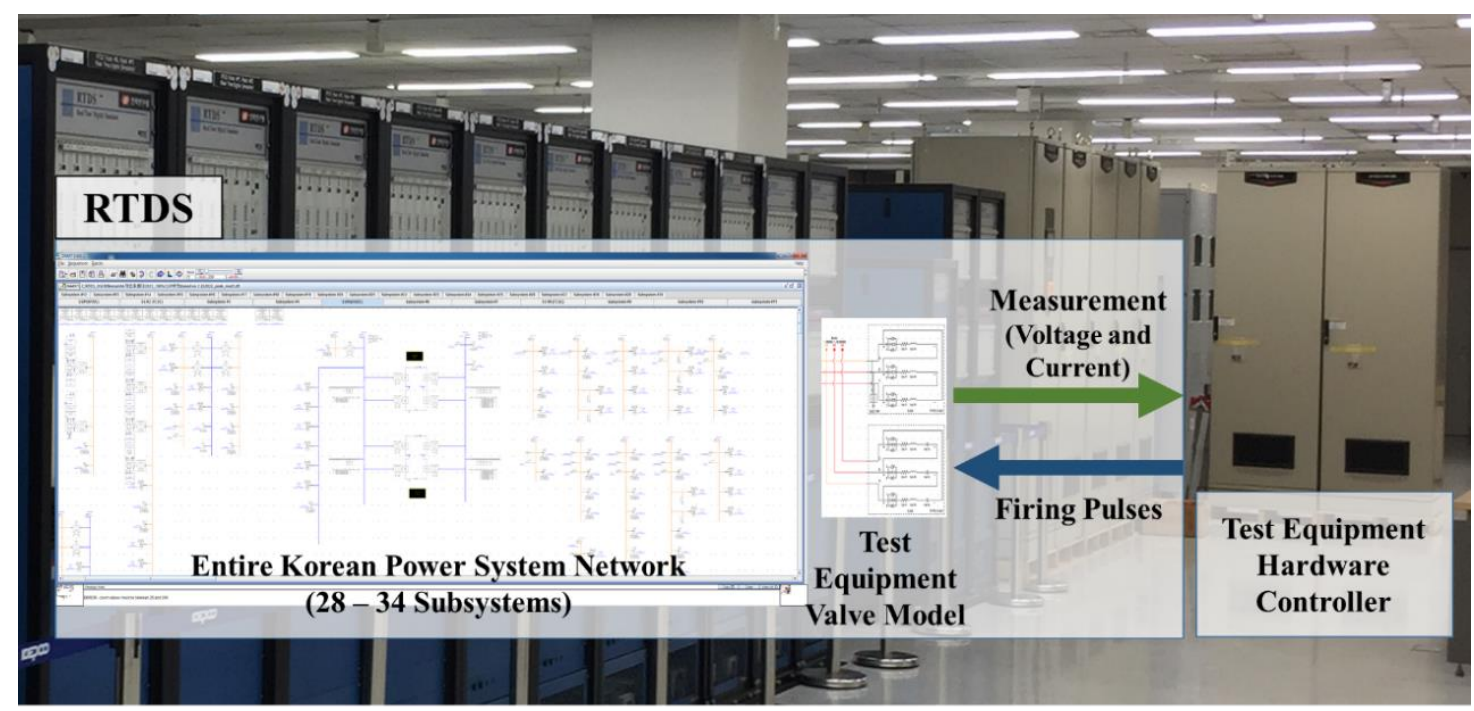

Figure 1. Configuration of dynamic performance test (DPT) for equipment.

Real-time Hybrid Simulator (RTHS) is a co-simulation tool that synthesizes RTS with transient stability analysis (TSA) tool to perform detailed real-time simulation of a large power system [2-5]. RTHS exchanges network power flow data of RTS and TSA program via the fiber-optic communication interface. A power system in RTHS is divided into electromagnetic transient subsystem in RTS and transient stability subsystem in the TSA program. The TSA program has the strength to build a wide area network, even though it lacks accuracy for power electronic equipment control and protection or interaction study. RTS has better accuracy of dynamic performance simulation for power electronics equipment. RTHS satisfies the accuracy of power electronics equipment dynamic performance in a large power system. Besides, RTHS with actual hardware controller and replicas can be used to study the interaction between power systems and power electronics equipment. Therefore, we will investigate the feasibility of HILS-DPT using RTHS with a practical case of Shin-Jecheon SVC compared to RTHS and large-scale RTS. 
The rest of the paper is organized as follows: We will present the definition, configuration, requirement, responsibility, and industrial experience of DPT in Section 2. The procedure, benefits, and boundary determination principle of HILS-DPT using RTHS are described in Section 3. As a representative case, HILS-DPT of the Shin-Jecheon SVC controller (+675/-225 MVar) using RTHS is presented in Section 4. Conclusions are presented in Section 5.

\section{HILS for Dynamic Performance Test in Large-Scale Power System}

\subsection{Definition and Features of HILS-DPT}

DPT was one of the processes in the factory acceptance test (FAT) conducted at the manufacturer's factory, which is carried out with equivalent or small size of the power system (e.g., 1 or 2 RTDS racks). DPT in FAT focuses on the dynamic response of the equipment itself. DPT in FAT cannot demonstrate the interaction between the equipment and the large AC power system. However, those studies' needs have been increased, and the number of electric utilities performing HILS-DPT with a large power system increases. We define that HILS-DPT is a test considering the dynamics of network and impact on adjacent power electronics equipment, which cannot be achieved in FAT and Site Acceptance Test (SAT). FAT has a limitation of test network scale, and SAT has an obstacle of available test items while in energization or operation of equipment on site. Therefore, HILS-DPT is a significant alternative to overcome those limits or constraints of FAT and SAT.

HILS-DPT evaluates equipment from a power system perspective. The requirements of HILS-DPT to accurately evaluate the role of equipment in a large power system are as follows:

- A sufficiently wide area of power system should be required to reflect the system dynamics and control characteristics of an adjacent power electronics equipment.

- A power system reliability study should be carried out whether it satisfies technical specifications though contingency analysis. Actual operation strategies and schemes (e.g., special protection scheme (SPS), emergency control) need to be applied.

- Even though the parameters were approved in FAT, the controller parameter can be changed upon request of electric utility based on the results of HILS-DPT.

Thus, HILS-DPT should be tested by the electric utility, due to an appropriate study network, the contingencies of the power system, and intellectual property issues (other manufacturers model and the replica controllers).

Figure 2 shows the procedure of HILS-DPT using large-scale RTS. The large power system is modeled in RTS using initial network data of the design stage in Step 1. Modeled large-scale power system interfaces with adjacent power electronics equipment models and other external hardware controllers (e.g., replica controller, relay, external system, etc.), and operation strategy is applied to test the power system model in Step 2. Functions, interaction, and contingency tests are conducted in Step 3. In Step 4, parameters are optimized at the network data for the operation stage. HILS-DPT evaluates and demonstrates its main role when the equipment works in the power system. 


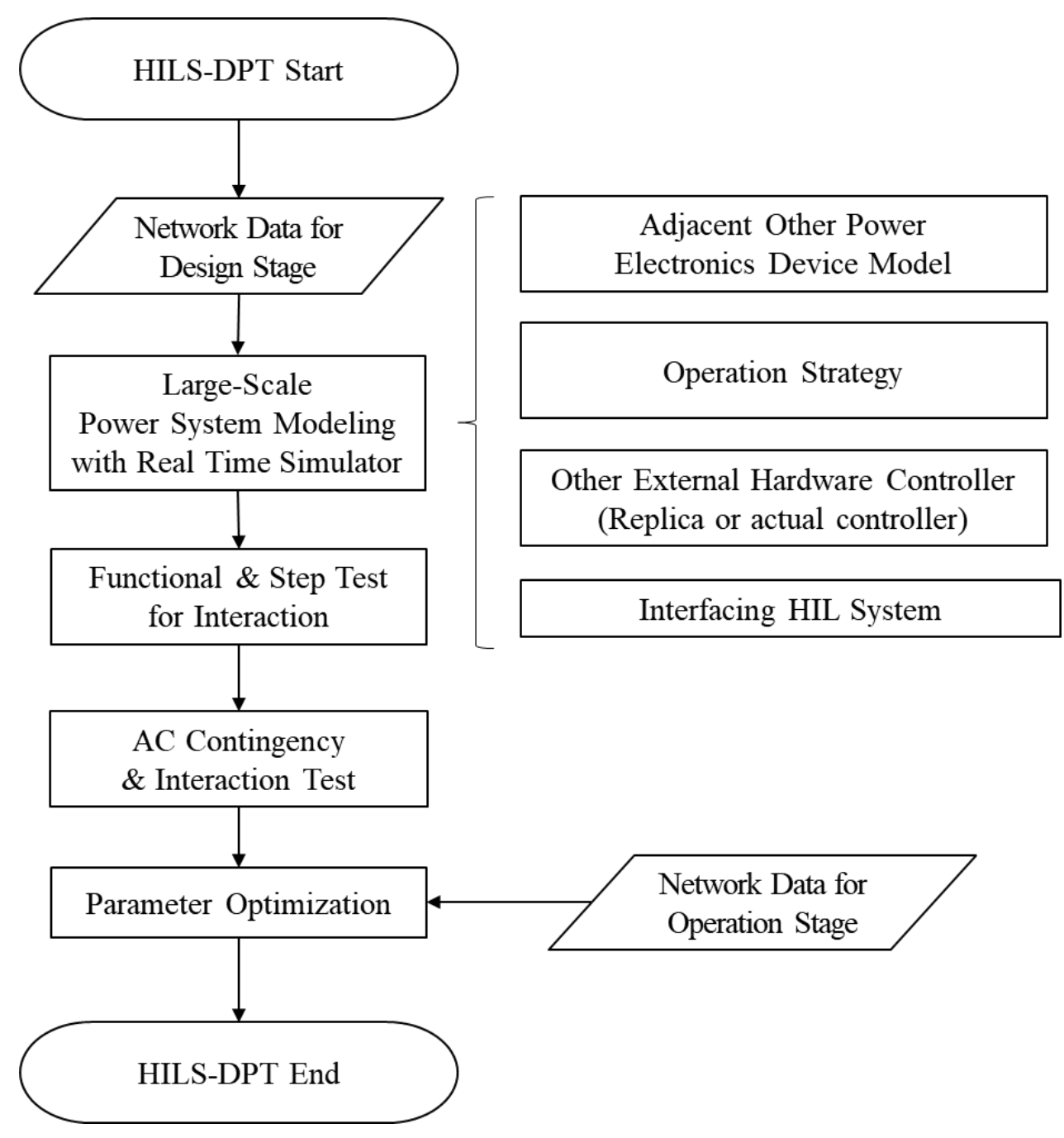

Figure 2. The entire procedure of hardware-in-the-loop simulation for dynamic performance test (HILS-DPT) using a large-scale real-time simulator (RTS).

\subsection{Responsibility, Experience, and Lessons of HILS-DPT}

HILS-DPT is necessary from an electric utility perspective, but it is quite a burdensome task for the manufacturer. This is because many other factors are affecting the condition of test equipment operation. For example, HILS-DPT with a large power system may be challenging to clarify the responsibility for the test results because network dynamics and adjacent power electronics devices can unintentionally distort the control characteristics of the test equipment. There may be an argument between an electric utility and manufacturer for the pass and fail, since it is difficult to evaluate quantitative criteria. Therefore, HILS-DPT is conducted as a witness test rather than an inspection test because the adjacent equipment and network are best known and responsible by the electric utility. HILS-DPT helps avoid the malfunctions that may occur during commissioning and commercial operation through the dynamic control performance test in the present system condition shortening the commissioning period by parameter tuning to the proper controller setting.

Figure 3 shows a practical case of parameter tuning for actual STATic synchronous COMpensator (STATCOM, 300 MVar, Godeok) controller. It is worth noting that HVDC (1.5 GW, Bukdangjin-Godeok, Line Commutated Converter) and STATCOM are installed at the same converter station. There are potential risks for interaction between HVDC and STATCOM. Before commissioning, HILS-DPT with a large-scale power system was conducted. The STATCOM step response considering HVDC operation at the same converter station is one of the test protocols for the interaction study. As shown in Figure 3, 
voltage, reactive power of STATCOM, and HVDC DC power had an oscillation and damped out slowly with initial parameter settings (blue line). STATCOM current controller gain was tuned, and the dynamic response of the STATCOM and HVDC was improved (as the green line in Figure 3). Figure 3 shows that the STATCOM controller was not well-tuned to present system conditions even though the controller setting was approved in FAT. Therefore, HILS-DPT is very useful during HVDC and FACTS projects, and its importance is to keep increasing.

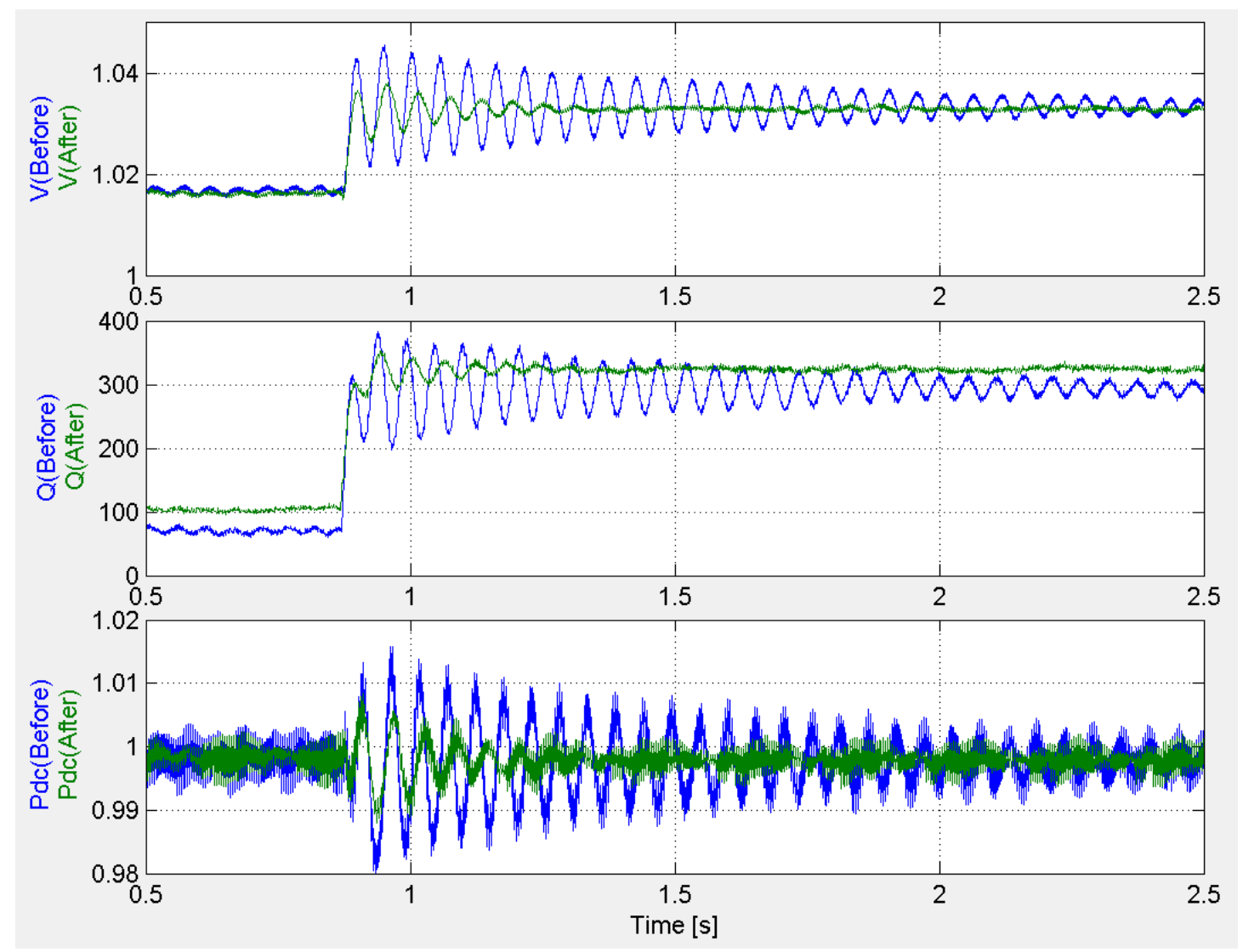

Figure 3. An example of parameter tuning experience during HILS-DPT; step-response test; voltage (first row), reactive power of STATCOM (second row), and active power of high-voltage direct current (HVDC; third row); before tuning (blue line) and after tuning (green line).

\section{HILS-DPT Using RTHS}

\subsection{Features of HILS-DPT Using RTHS}

A hybrid simulation is a powerful tool because power electronics applications in the power system require detailed studies with Electro-Magnetic Transient (EMT) simulation investigating the performance of the equipment, and electric utility also has needs of dynamic investigation in the large power system [3-16]. Although many hybrid simulation methods and tools exist, improving the accuracy and efficiency of a large power system simulation, those hybrid simulation tools are based on non-real-time. KEPCO, Powertech Lab, and Yonsei Univ. jointly developed RTHS combining the RTS and TSA program because there are needs of testing replicas in the KEPCO advanced real-time simulation laboratory to investigate the future power system issues [2-6]. Figure 4 shows the configuration of RTHS. RTHS has following features for HILS-DPT:

1. Wide network dynamics

The simulation case network using RTHS is divided into the network simulated with the RTS as the internal subsystem and the remaining network simulated with the TSA as the external 
subsystem. Wide area AC network dynamics, including hundreds of generators and transmission lines, can be modeled in an external subsystem. This allows us to study the system-wide impact efficiently and effectively.

2. Flexibility

The flexibility of the simulation study is enhanced because the external subsystem network topology can easily be modified with the TSA program. Various load conditions (such as peak and off-peak cases) and models (such as dynamic load model, composite load model, and constant load model), contingencies, operating points, and strategies can be applied in the external subsystem unless internal subsystem has a significant change of network topology. The dynamic load model is especially difficult to apply to large-scale RTS, due to the numerical instability and the highly required computational hardware resources. Whereas, RTHS can apply for a dynamic load model in the entire power system with the TSA program, and the power system characteristics using a dynamic load model becomes more accurate than those using a constant load model. Thus, RTHS with a dynamic load model enables the achievement of a more accurate response of power system and power electronics equipment.

3. Economical advantage

Large-scale RTS requires more than 28 racks to simulation the entire Korean power system, and the future power system will be larger and more complicated. Expanding RTS more and more is not an economical solution for future power system studies. From the KEPCO experience since 2016, RTHS requires five or fewer racks to simulate the entire Korean power system, and RTHS is free for the power system expanding issue.

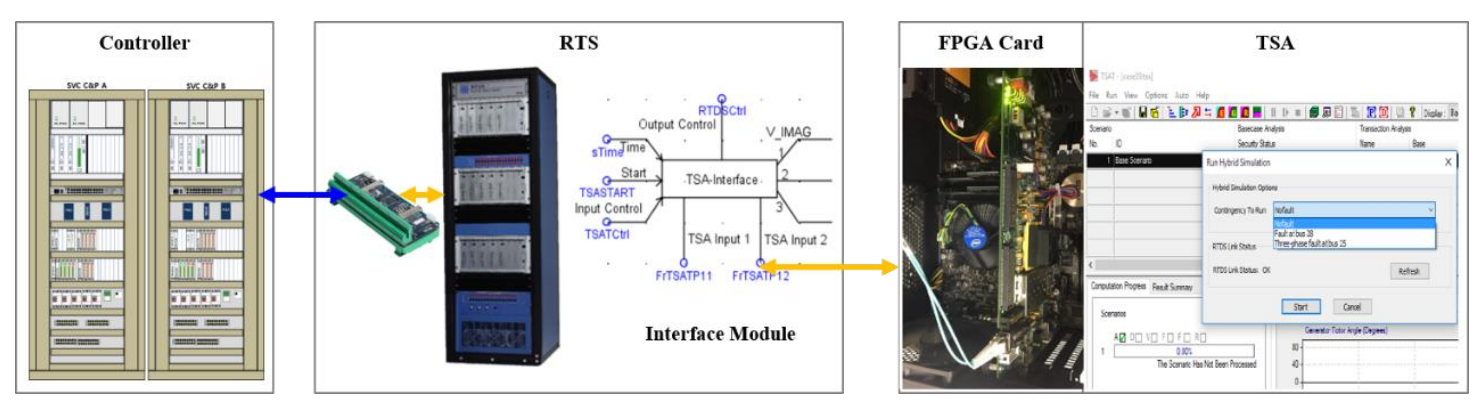

Figure 4. Configuration of real-time hybrid simulator (RTHS).

\subsection{The Procedure of RTHS for HILS-DPT}

Figure 5 shows the procedure of RTHS. The biggest difference between simulation using a large-scaled RTS and simulation using RTHS is the boundary area. RTHS cannot be relied upon unless the boundary buses are properly set. Setting up the boundary busses is the first step of the HILS-DPT using RTHS, and the boundary bus determination method for this practical SVC HILS-DPT will be presented in Section 3.3. The second step is the extraction of an internal and external area with TSA network data. Then, we can get two TSA network data; internal subsystem for RTS and external subsystem for TSA. In the next step, internal subsystem network data is converted to the RTS model. This RTS model includes only transmission lines, transformers, generators, and loads. Then, power electronics equipment and communication interface models are added to the RTS model manually. We call this step Power System Modeling (RTS) in Figure 5.

After configuring the base case of RTS model of an internal subsystem, various RTHS case with scenarios needs to be developed. In this step, an external hardware controller like a replica is implemented for HILS-DPT. When test equipment replica or actual controller is connected to RTS, HILS-DPT using RTHS is ready. All power electronics equipment in the RTS area must run at an operating point based on the study network data before releasing the locks of the generator governor. 
Although the power flow on the boundary buses must be the same as TSA power flow, power flow mismatch between internal subsystem simulation and TSA power flow can occur due to the difference of the basic computation algorithm of the two different tools. In our practical case of SVC HILS-DPT, the mismatch was less than $100 \mathrm{MW}$. If the mismatch is greater than $1 \mathrm{GW}$, initialization failed. Once the simulation setup is completed, HILS-DPT using RTHS can be performed. If an abnormal operation of a controller or protection functions that were not found in FAT or interaction between power electronics equipment and power system is found, the root cause must be analyzed and corrected.

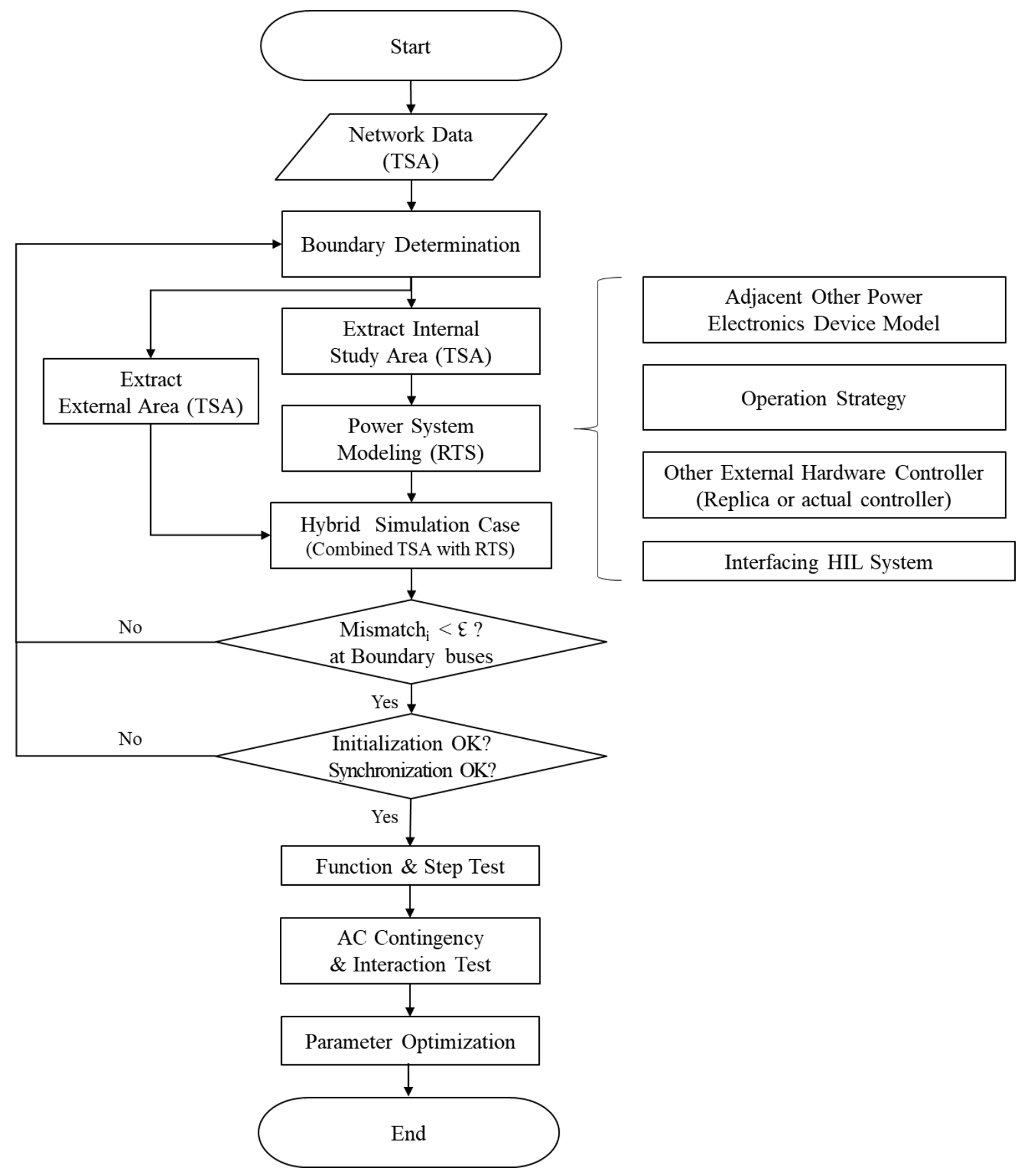

Figure 5. The overall procedure of RTHS for HILS-DPT. 


\subsection{Determination of Boundary Area of RTHS for HILS-DPT}

The boundary buses can be set depending on the condition of the power system, fault location, fault type, and interest study area. During the RTHS development from 2016 through 2018, we concluded that RTHS requires at least three RTDS racks with six PB processor cards per rack for the internal area of the entire Korean power system to achieve a similar response of a large-scale RTS. In our experience, 4-6 racks of RTDS are required to perform HILS-DPT using RTHS under a large-scale power system. It is worth noting that it is better to set a wide internal area in the RTHS. The internal area determination algorithm based on the electrical distance between boundary buses and fault location is introduced in $[9,10]$. Although the wide internal area of the RTHS offers similar simulation results to the large-scale RTS, it reduces the benefits of the hybrid simulation. On the contrary, the narrow internal area of the hybrid simulation decreases accuracy. Thus, the following boundary determination principles of RTHS are applied in Shin-Jecheon SVC HILS-DPT.

1. Select boundary buses with an electrical distance of more than a certain threshold (0.4) from the bus with the most serious fault $(765 \mathrm{kV}$ transmission line fault).

2. Select boundary buses with an electrical distance of more than a certain threshold (0.4) from the power electronics equipment, if the power electronics are nearby the boundary.

3. Select the boundary buses without island areas.

4. Include the generators in the RTS area, if there are the generators around the boundaries.

5. Determines the number of hardware racks of the RTS system, satisfying the above conditions.

6. Expand the RTS area as wide as possible.

The flow chart of the boundary determination method for SVC HILS-DPT using RTHS is shown in Figure 6.

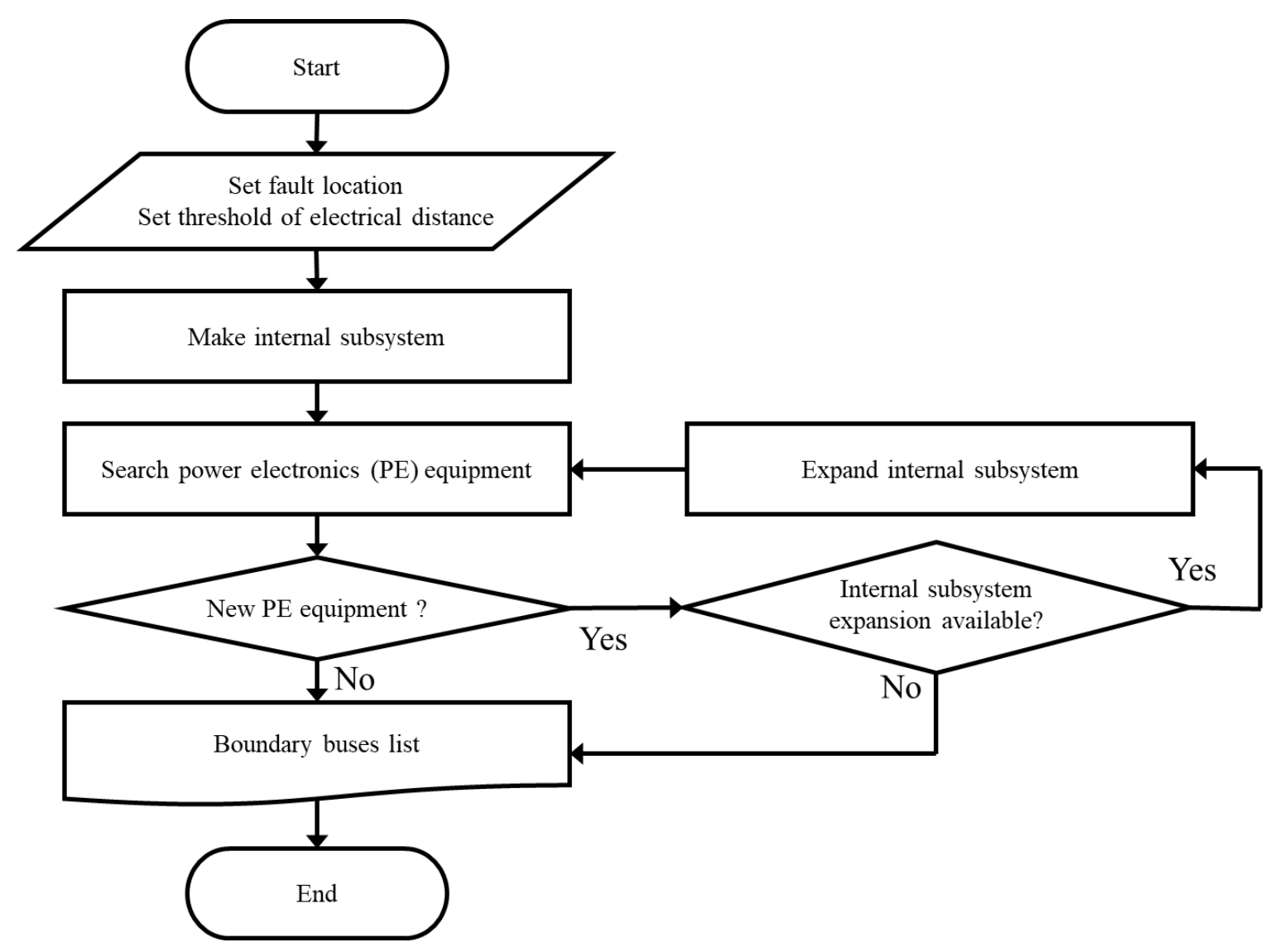

Figure 6. The procedure of determination of boundary buses. 
The electrical distance $\left(D_{i j}\right)$ between $i$ bus and $j$ bus is defined as following (1) [10]

$$
D_{i j}=-\log _{10} \frac{\left(\frac{\delta V}{\delta Q}\right)_{i j}}{\left(\frac{\delta V}{\delta Q}\right)_{j j}}=-\log _{10} \frac{\Delta V_{i}}{\Delta V_{j}}
$$

$\Delta V_{i}$ is voltage sensitivity at $i$ bus, and $\Delta V_{j}$ is voltage sensitivity at $j$ bus where the reactive power $(Q)$ changes at $j$ bus. The electrical distance is calculated based on the voltage sensitivity derived from QV part of the Jacobian matrix of the power system.

\section{A Practical Application of HILS-DPT Using RTHS; Shin-Jecheon SVC HILS-DPT}

\subsection{Background and RTHS Case Set Up}

The eastern part of the Korean power system is designed to transfer electric power from a huge nuclear power plant to the load center. Total power generation from nuclear power plants is $8 \mathrm{GW}$, and $345 \mathrm{kV}$ and $765 \mathrm{kV}$ transmission lines transmit those power to a metropolitan area near Seoul. Because $5.4 \mathrm{GW}$ of power is transmitted through the $765 \mathrm{kV}$ transmission lines during peak case, the contingency of $765 \mathrm{kV}$ transmission lines might threaten the stability of the entire power system. To avoid system instability, KEPCO planned to install multiple power electronics equipment around the $765 \mathrm{kV}$ and $345 \mathrm{kV}$ transmission lines. Shin-Jecheon SVC is among those facilities, and it is installed to improve voltage stability of $345 \mathrm{kV}$ transmission line in 2018. Note that HILS-DPT of Shin-Jecheon SVC using RTHS is the first case of practical use of RTHS.

Table 1 presents one of the contingency scenarios for HILS-DPT of SVC, which tests $765 \mathrm{kV}$ transmission line fault and following reclose failure. Table 2 shows the power electronics equipment location, control mode, operating point, and ratings in the test case and its modeling area. Because the HVDC is far from the fault locations and the test equipment, it is modeled in the external subsystem (TSA program). Because the rest of the power electronics equipment is located nearby the SVC, they are modeled in the internal subsystem (RTS). Figure 7 illustrates the case study map, and the internal subsystem details and simulation settings are listed in Table 3. The actual special protection scheme for $765 \mathrm{kV}$ transmission line fault is applied to HILS-DPT. When $765 \mathrm{kV}$ fault occurs, four Thyristor Controlled Series Compensator (TCSC)installed at the $345 \mathrm{kV}$ transmission line boost their compensation level from $50 \%$ to $70 \%$. As power flows through the $345 \mathrm{kV}$ transmission lines increases, SVC and STATCOMs respond to compensate for the voltage drop.

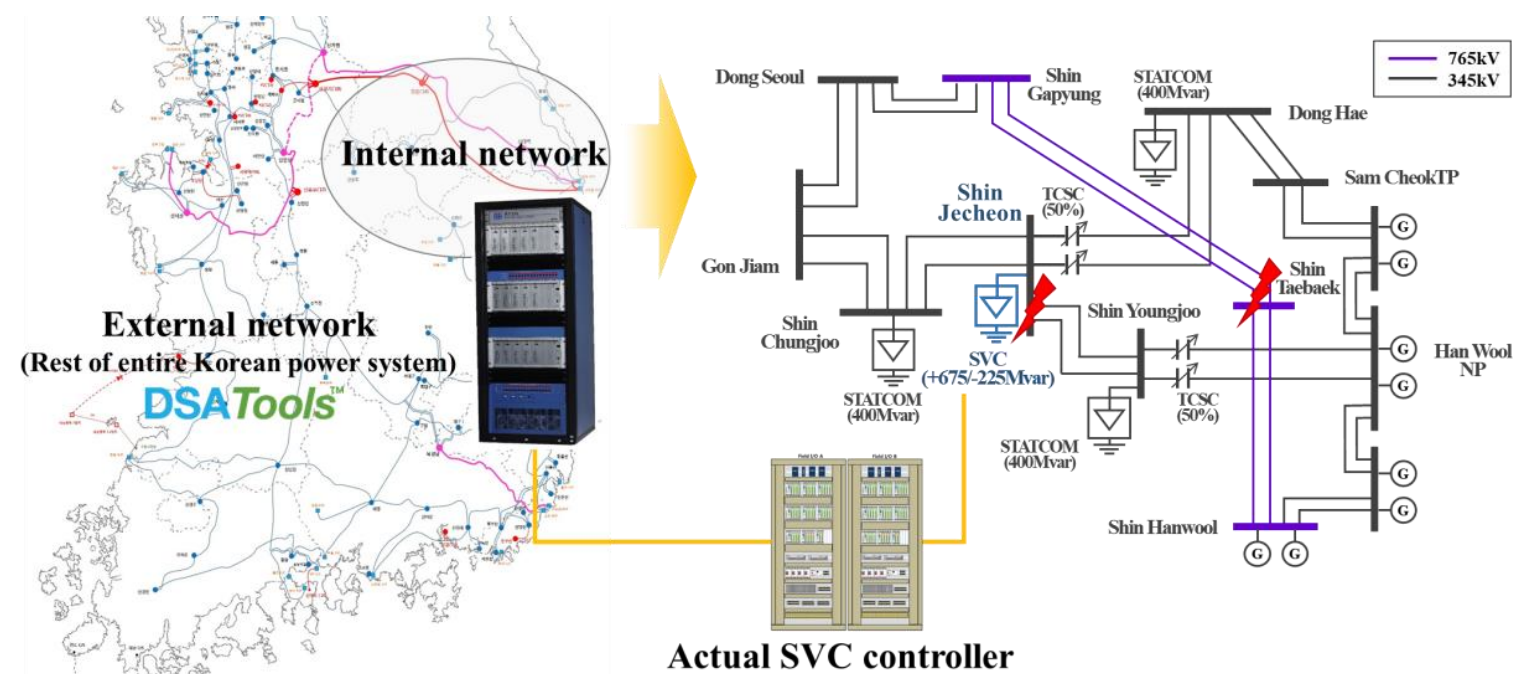

Figure 7. Case study map of Shin-Jecheon SVC HILS-DPT using RTHS. 
Table 1. Contingency scenario for $765 \mathrm{kV}$ transmission line fault.

\begin{tabular}{cc}
\hline Time (Cycle) & Action \\
\hline 0 & Fault occurs \\
\hline 5 & $\begin{array}{c}765 \mathrm{kV} \text { line circuit breaker open } \\
\text { TCSC compensation boost }(50 \% \rightarrow 70 \%)\end{array}$ \\
\hline 9 & Generator Trip $(1.5 \mathrm{GW})$ \\
\hline 71 & $765 \mathrm{kV}$ line reclose attempted \\
\hline 76 & Reclose failure \\
\hline
\end{tabular}

Table 2. Power electronics equipment in the test case. SVC, Static VAR Compensator.

\begin{tabular}{cccc}
\hline Type (Modeling Region) & Location & Control Mode (Setpoint) & Ratings (Number of Equipment) \\
\hline HVDC (External) & Bukdangjin Godeok & Power control (1.0 p.u.) & $1.5 \mathrm{GW}$ \\
\hline \multirow{2}{*}{ TCSC (Internal) } & Sinyoungju & Impedance control (50\%) & $555 \mathrm{MVar}(2)$ \\
\cline { 2 - 4 } & Shin-Jecheon & Impedance control (50\%) & $595 \mathrm{MVar}(2)$ \\
\hline \multirow{3}{*}{ STATCOM (Internal) } & Donghae & Q reserve control (1.0 p.u.) & $\pm 400 \mathrm{MVar}$ \\
\cline { 2 - 4 } & Sinyoungju & Q reserve control (1.0 p.u.) & $\pm 400 \mathrm{MVar}$ \\
\cline { 2 - 4 } & Sinchungju & Q reserve control (1.0 p.u.) & $\pm 400 \mathrm{MVar}$ \\
\hline SVC (Internal) & Shin-Jecheon & Q reserve control (1.0 p.u.) & $+675 /-225 \mathrm{MVar}$ \\
\hline
\end{tabular}

Table 3. Simulation environment details of RTHS and the large-scaled RTS for Shin-Jecheon SVC HILS-DPT.

\begin{tabular}{ccc}
\hline Types & RTHS & Large-Scaled RTS \\
\hline Number of RTDS rack & 4 & 29 \\
\hline Number of bus & 103 & 1140 \\
\hline Equipment & 8 & 10 \\
& $(1$ SVC, 3 STATCOMs, 4 TCSCs) & $(1$ HVDC, 1 SVC, 4 STATCOMs, 4 TCSCs) \\
\hline Generators & $12(11.12$ GW $)$ & $228(87.5$ GW $)$ \\
\hline Time step & $75 \mu \mathrm{sec}(\mathrm{RTS}), 4$ msec (TSA Program) & $75 \mu \mathrm{sec}$ \\
\hline
\end{tabular}

The test is conducted by a large-scale RTS and RTHS to investigate the feasibility of RTHS applying for HILS-DPT. The large-scale RTS needs 29 subsystems, with six PB5 processor cards for each subsystem. RTHS uses four subsystems for the internal subsystem, and TSA program covers the rest of the power system. The internal area of RTHS includes detailed modeling of large power generators, transmission lines, STATCOMs, TCSCs, and SVC. During the development of the RTHS, we compared the Korean power systems with the RHTS and Full-RTDS and found that the results of the hybrid simulation are reliable when the electrical distance between boundary buses and the fault location is 0.4 or less in the Korean power system. Thus, the threshold for determining the boundary buses was set to 0.4 empirically, but this depends on the power system condition, types of contingencies, and HVDC and FACTS location. The energy data for each boundary bus is exchanged through fiber optic communication cable $[4-6,12,13]$. This test case has 17 boundary buses. SVC actual controller is shown in Figure 8. The list of DPT and contingency list of HILS-DPT are shown in Appendix A. 


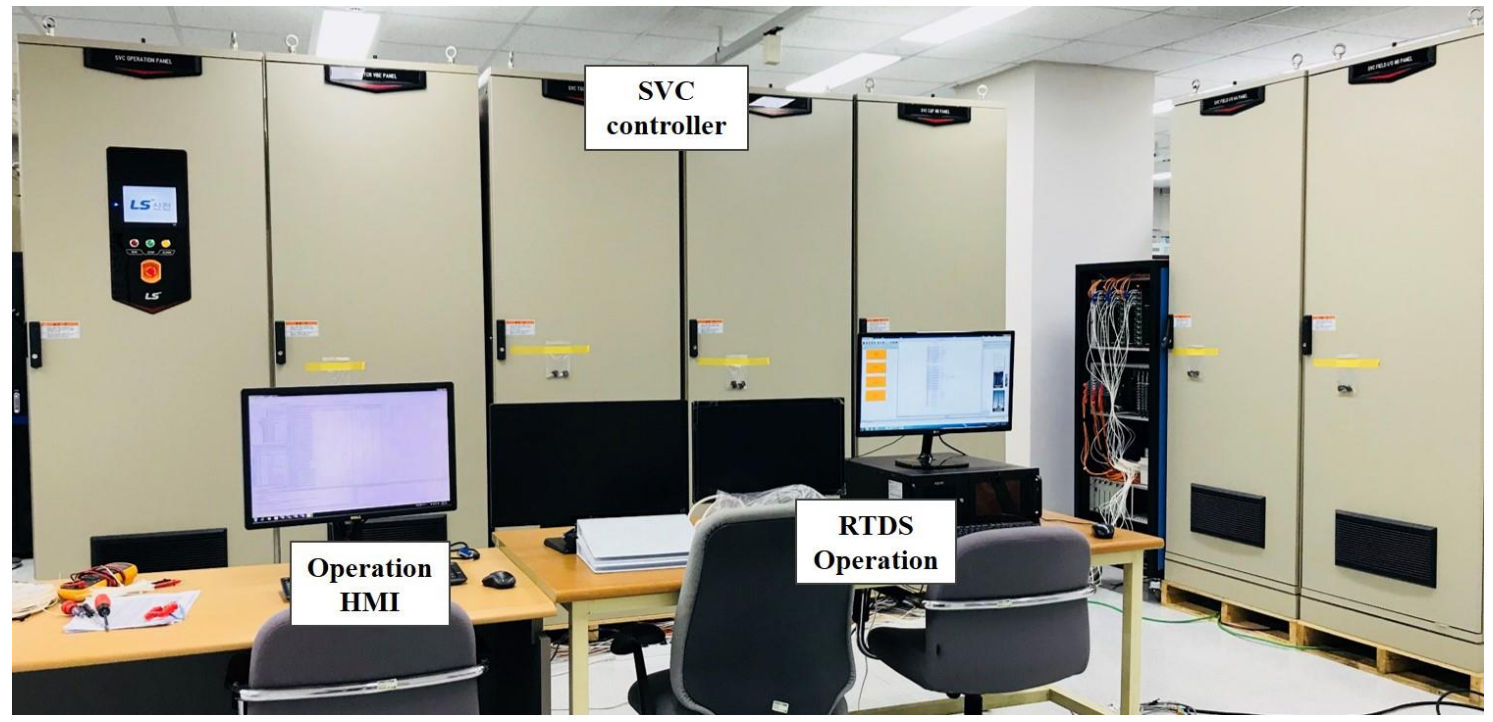

Figure 8. SVC controller interfaced with RTHS.

\subsection{Results of HILS-DPT Using RTHS and Large-Scale RTS}

This section presents the simulation results of HILS-DPT using RTHS and large-scale RTS to investigate the feasibility of RTHS applying HILS-DPT.

- $\quad$ Case 1: A $345 \mathrm{kV}$ line fault (3-phase to ground)

The 3-phase to ground fault is applied to Shin-Jecheon bus during six cycles at $0.5 \mathrm{~s}$. During the fault, the bus voltage drops to zero and SVC could not compensate it. After the fault is cleared, the bus voltage recovers immediately, and the reactive power output of SVC increased according to network recovery characteristics. Overall, the SVC dynamic performance of RTHS is almost the same as HILS-DPT using large-scale RTS. Figure 9 shows the bus voltage at Shin-Jecheon and reactive power of SVC of RTHS simulation and RTS simulation when 3-phase fault is applied. A small difference of less than 10 MVar of SVC reactive power dynamics between RTHS and RTS simulation can be seen in Figure 9. Even though the bus voltage has a small difference of less than 0.01 p.u., SVC responses to the voltage and reactive power output are slightly different from each other. The authors concluded that this amount of difference is acceptable, and HILS-DPT using RTHS is feasible.

- Case 2: A $345 \mathrm{kV}$ unbalanced fault (single line to ground)

The A-phase to ground fault was applied to Shin-Jecheon bus during six cycles at $0.5 \mathrm{~s}$. During the fault, the bus voltage drops to around 0.9 p.u. Figure 10 shows the bus voltage at Shin-Jecheon and reactive power of SVC of RTHS simulation and RTS simulation when a single-phase fault is applied. Even though unbalanced fault is applied at Shin-Jecheon bus, the bus voltage at Shin-Jechoen and reactive power output of SVC is almost the same. The authors concluded that this amount of difference is acceptable, and HILS-DPT using RTHS is feasible.

- Case 3: A $765 \mathrm{kV}$ line fault (3-phase to ground), generator trip SPS, and emergency control

The $765 \mathrm{kV}$ transmission double line fault has complicated sequences, such as TCSC boost up and nearby generators trip, as shown in Figure 11. Moreover, its impact on the network is larger than any other contingencies. The faulted location is just 3-level away from Shin-Jecheon bus, where the SVC is installed. When the fault occurred at $0.5 \mathrm{~s}$, the Shin-Jecheon bus voltage dropped to around 0.7 p.u., and SVC fully outputs reactive power to compensate for the low voltage. After the fault cleared, it recovered to a lower limit of voltage deadband and swing according to the network dynamics. Figure 13 shows the bus voltage at Shin-Jecheon and reactive power of SVC of RTHS simulation and RTS simulation when 3-phase fault is applied at $765 \mathrm{kV}$ transmission 
lines. A small difference of less than 10 MVar of SVC reactive power dynamics between RTHS and RTS simulation can be seen in Figure 13. Even in this case, HILS-DPT using RTHS and RTS have almost the same dynamic. Therefore, the authors concluded that this amount of difference is acceptable, and HILS-DPT using RTHS is feasible.

- Case 4: A $765 \mathrm{kV}$ line fault (3-phase to ground), generator trip SPS, and emergency control; dynamic load model applied

As mentioned in the previous section, RTHS has the strength of flexibility for modeling of the external subsystem. For RTS simulation, it is not easy to apply various load models to the entire network, due to its computational burden. However, it depends on the study purpose; consideration of the various load models (e.g., induction motors and ZIP) in the external subsystem could be required. Figure 12 shows the result for SVC responses using RTHS when different the load model is applied, and $765 \mathrm{kV}$ 3-phase fault occurs. The simulation results of the constant impedance load model (blue line) and induction motor and ZIP load model (green line) are shown in Figure 12. Because the induction motor and ZIP load model absorb more reactive power than the constant impedance load model, the voltage at Shin-Jechoen bus in Figure 12 shows that voltage drop with induction motor and ZIP load model is higher than voltage drop with constant impedance load model. This analysis might be important in future power system studies. As can be seen in Figure 12, the flexibility of the external subsystem will be great advantages of RTHS for HILS-DPT.

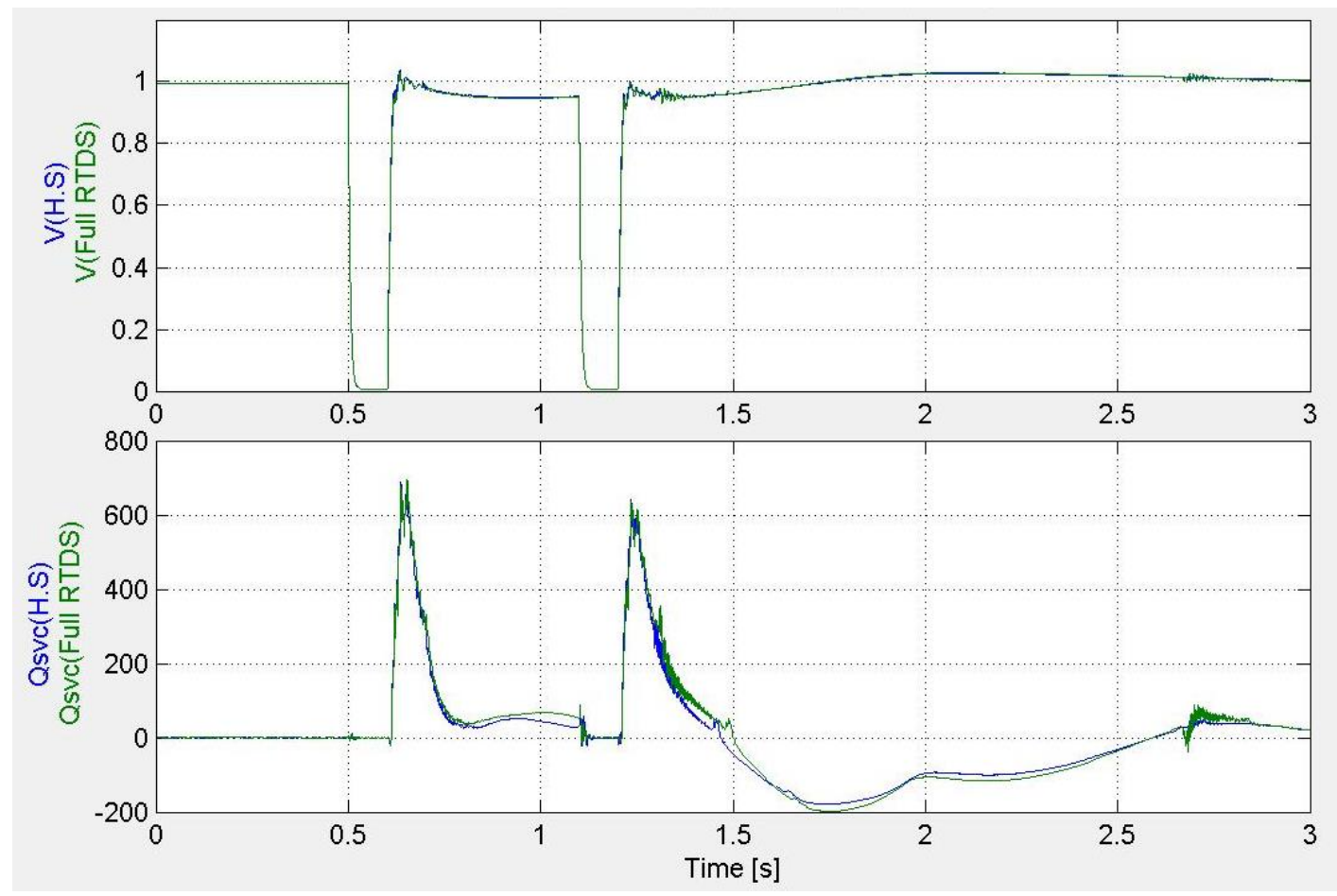

Figure 9. A $345 \mathrm{kV}$ line fault is applied at Shin-Jecheon bus; Shin-Jecheon bus voltage (first row) and reactive power of SVC (second row); RTHS (blue line) and large-scale RTS (green line). 


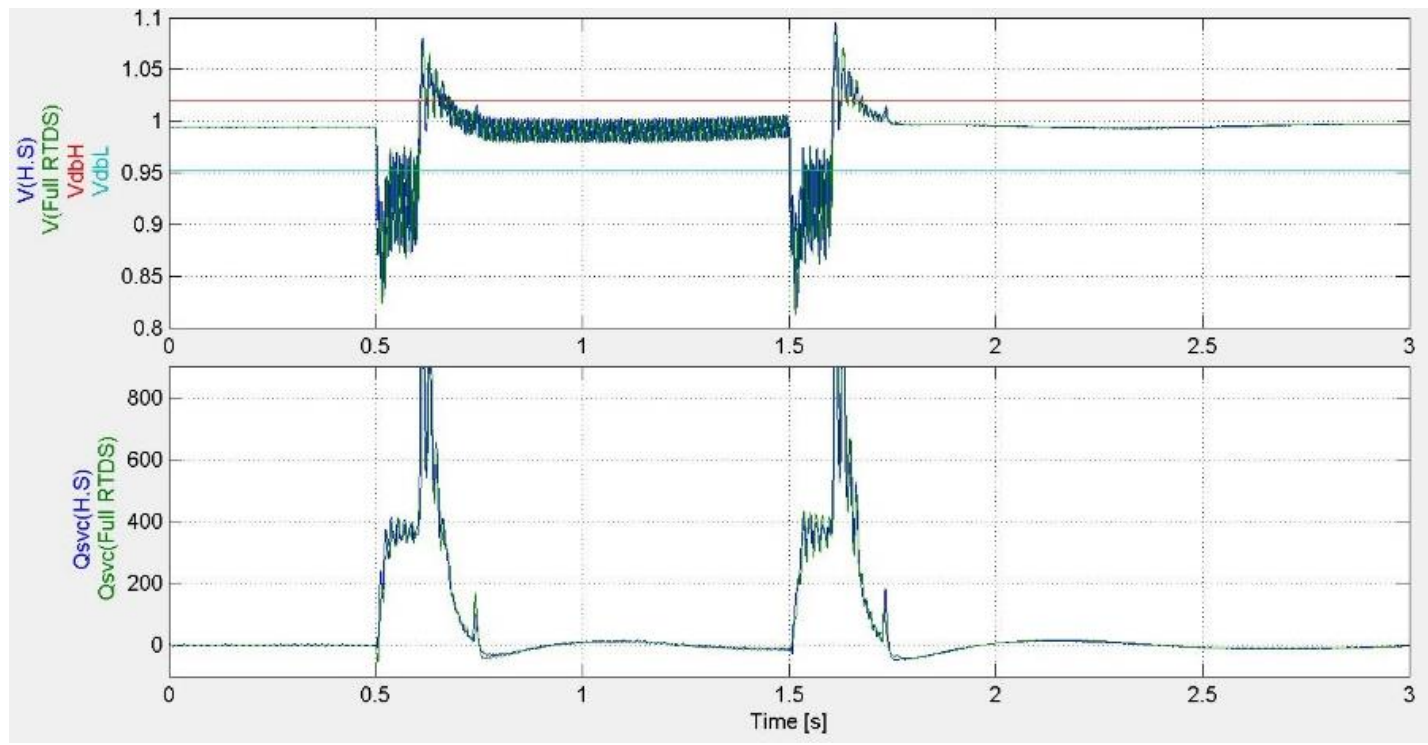

Figure 10. A $345 \mathrm{kV}$ line unbalanced fault is applied at Shin-Jecheon bus; Shin-Jecheon bus voltage (first row) and reactive power of SVC (second row); RTHS (blue line) and large-scale RTS (green line)

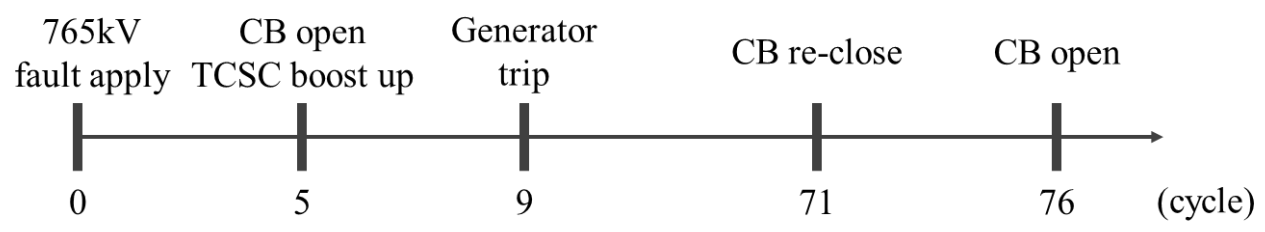

Figure 11. $765 \mathrm{kV}$ line fault sequence considering emergency control and SPS.

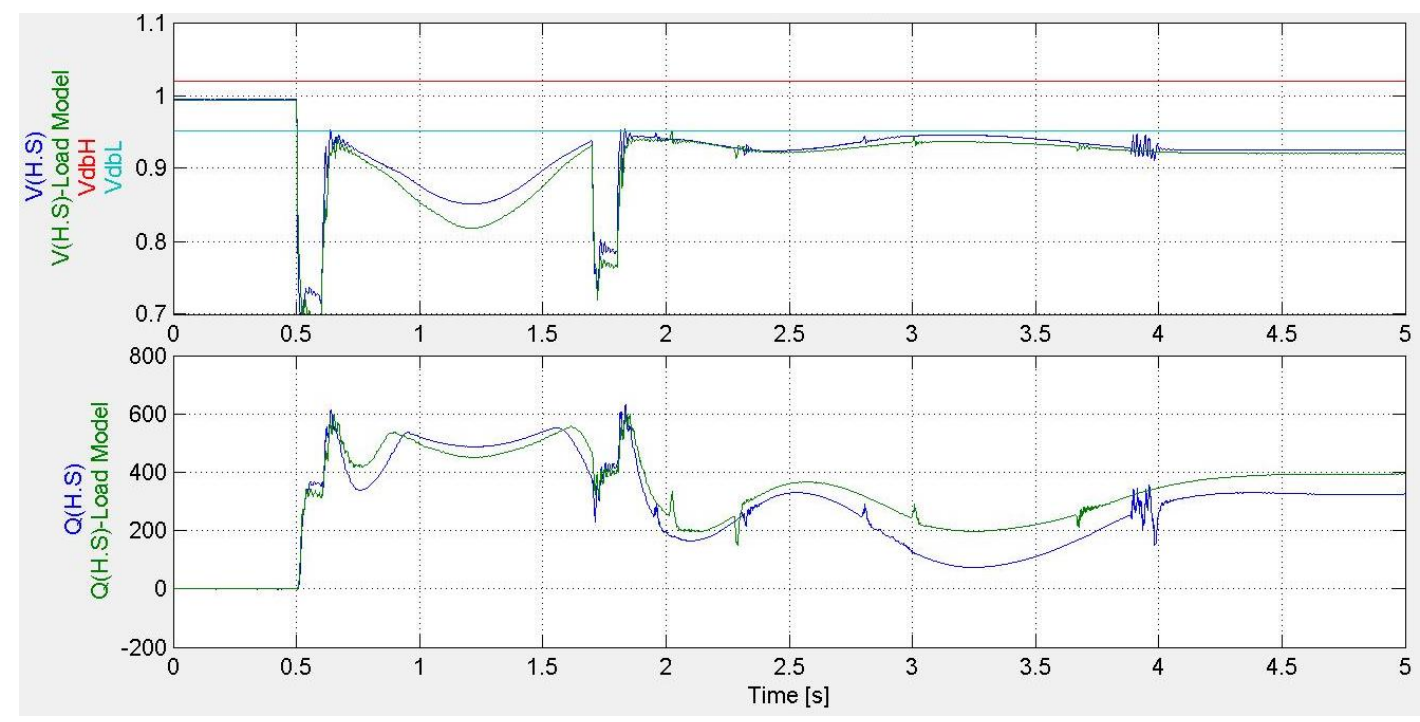

Figure 12. A $765 \mathrm{kV}$ line fault nearby Shin-Jecheon bus considering induction motor and ZIP load model; Shin-Jecheon bus voltage (first row) and reactive power of SVC (second row); RTHS (blue line) and large-scale RTS (green line). 


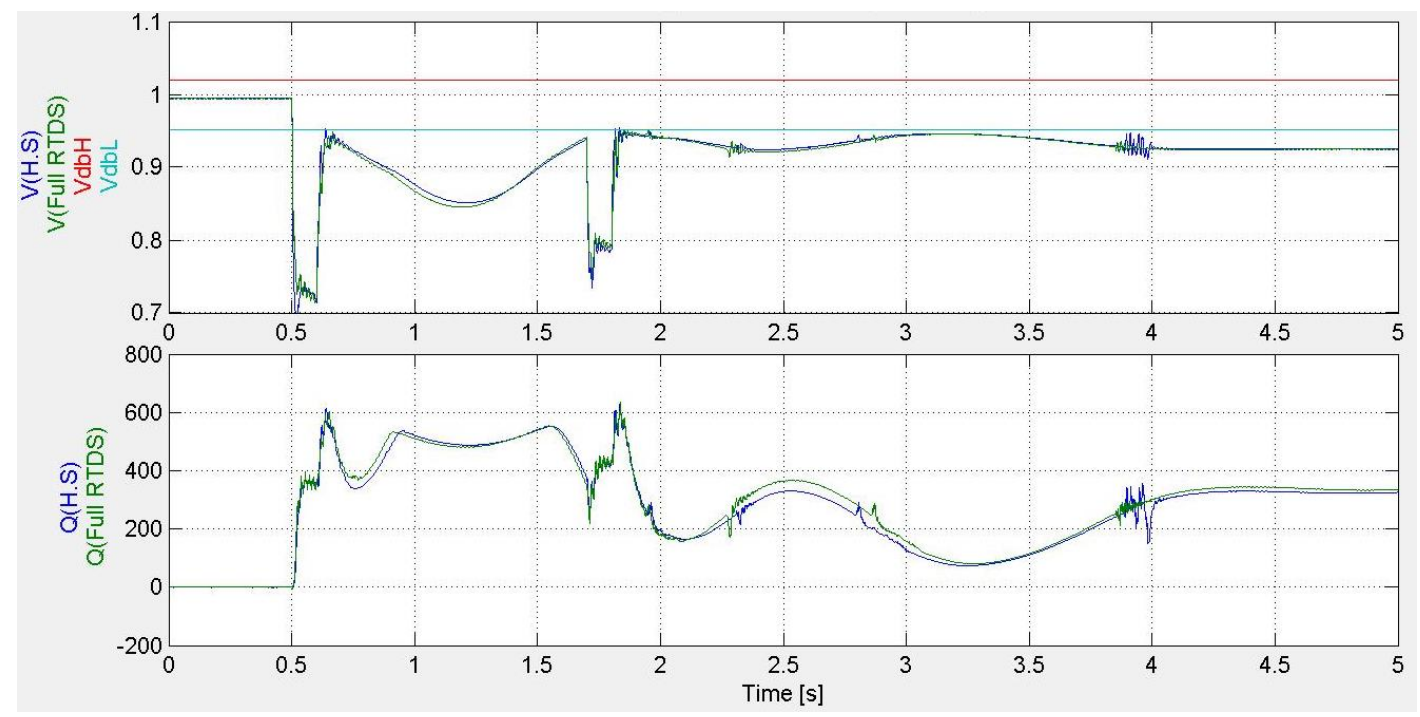

Figure 13. A $765 \mathrm{kV}$ line fault is applied nearby Shin-Jecheon; Shin-Jecheon bus voltage (first row) and reactive power of SVC (second row); RTHS (blue line) and large-scale RTS (green line).

\section{Conclusions}

As the complexity of the modern power system grows, power system simulation and studies need to be flexible to investigate various operating points and conditions. Although a large-scale power system modeling and simulation are useful, it is less flexible than hybrid simulation as power systems become more and more complicated, and as renewable energy resources increase. That is the reason that hybrid simulation and co-simulation has been developed and expand their applications [4-18]. Hybrid simulation improves accuracy because equivalents, which represent areas of non-interest in the power system, do not completely show the responses of the actual power system.

Real-time Hybrid Simulator supports system-wide impact study efficiently and effectively because hundreds of generators and transmission lines can be modeled in the TSA program. This improves the flexibility of the simulation study because the network topologies and load conditions in TSA subsystem (which is called the external subsystem in this paper) can easily be modified. RTHS is free for the power system expanding issue for the future power system. RTHS can be used in replica test applications with real-time attributes. HILS-DPT, which test replica or actual controller of high-voltage power electronics equipment, is one of the best applications of RTHS.

HILS-DPT using RTHS validates the controls and operation of newly installed power equipment and shows its impact on the power system before its installation. It is worth noting that HILS-DPT shortens the duration of SAT and ensures SAT progress smoothly, helping utilities take over the equipment. HILS-DPT shows and validates the response of the equipment during the contingencies that cannot be performed on FAT and SAT. This improves the reliability of the equipment and allows the utility to have confidence in the equipment. In particular, HILS-DPT using RTHS can apply the saved hardware resources to the dynamic load model, while yielding similar results to the actual power system. The saved hardware resources can be used for the renewable energy source model, which is an important factor in the future power system. This will allow the interaction and influence between power electronics equipment planned to be newly installed and renewable energy sources to be studied. The authors investigated the feasibility of HILS-DPT using RTHS, and concluded RTHS is applicable for HILS-DPT. KEPCO will continue to apply more HVDC and FACTS replica controllers, and further research and development are being conducted related to its operation, and analysis technology, and RTHS will help the engineers perform tests and studies efficiently and effectively. 
Author Contributions: Methodology, all authors; Software, all authors; writing—original draft preparation, J.S. (Jiyoung Song), K.H., J.L. (Jeehoon Lee), H.L., and H.K.; writing-review and editing, J.S. (Jiyoung Song), K.H., and H.K.; supervision, K.H. and H.K.; project administration, J.S. (Jeonghoon Shin). All authors have read and agreed the published version of the manuscript.

Funding: This work was supported by "Human Resources Program in Energy Technology" of the Korea Institute of Energy Technology Evaluation and Planning (KETEP) granted financial resource from the Ministry of Trade, Industry \& Energy, Republic of Korea. (No.20194030202420) This research was also supported by Korea Electric Power Corporation (Grant number: R20XO02-4).

Conflicts of Interest: The authors declare no conflict of interest.

\section{Appendix A}

The list of DPT is as follows:

- $\quad$ Start and stop sequences test

- Control modes test

- $\quad$ Emergency stop test

- Continuous operation test

- $\quad$ Protection test (overvoltage, under-voltage, under frequency, and internal fault)

- Mode change test

- Dynamic response test during an AC fault

- Verification of black-box model

The contingency list of HILS-DPT is as follows:

- $\quad 765 \mathrm{kV}$ transmission line trip with TCSC boost up and generator trip for special protection scheme (2 cases)

- $\quad 345 \mathrm{kV}$ transmission line trip (1 case)

- $\quad$ Single-phase fault and line fault at $345 \mathrm{kV}$ transmission line (1 case for each)

- $\quad$ TCSC bypass (1 case)

- $\quad$ STATCOM trip (3 cases)

- $\quad$ Generator trip (1 case)

\section{References}

1. Kim, H.; Kim, J.; Song, J.; Lee, J.; Han, K.; Shin, J.; Kim, T.; Hur, K. Smart and Green Substation Shaping the Electric Power Grid of Korea. IEEE Power Energy Mag. 2019, 17, 16-24. [CrossRef]

2. Kim, S.; Kim, H.; Lee, H.; Lee, J.; Lee, B.J.; Jang, G.; Lan, X.; Kim, T.; Jeon, D.; Kim, Y.; et al. Expanding power systems in the Republic of Korea feasibility studies and future challenges. IEEE Power Energy Mag. 2019, 17, 61-72. [CrossRef]

3. Hur, K.; Lee, J.; Song, J.; Ko, B.; Shin, J. Advanced Real Time Power System Simulator for Korea Electric Power Systems: Challenges and Opportunities. Presented at IEEE PES, Portland, OR, USA, August 2018; Available online: http://site.ieee.org/pes-itst/files/2018/08/2018-Panel-1.pdf (accessed on 25 July 2020).

4. Lin, X.; Zadehkhost, P.; Lee, J.; Song, J.; Ko, B.; Hur, K. TSAT-RTDS Interface-The Development of a Hybrid Simulation Tool. Presented at IEEE PES, Chicago, IL, USA, July 2017; IEEE PES. 2017. Available online: http://site.ieee.org/pes-itst/files/2018/08/2018-Panel-1.pdf (accessed on 25 July 2020).

5. RTDS Technologies Inc. IEEE PES. 2018. Available online: https://knowledge.rtds.com/hc/en-us/articles/ 360034295014-TSAT-RTDS-Interface/ (accessed on 25 July 2019).

6. Powertech Labs Inc. IEEE PES. 2018. Available online: https://www.dsatools.com/tsat-rtds-interface-tri/ (accessed on 25 July 2020). 
7. Park, I.K.; Lee, J.; Song, J.; Kim, Y.; Kim, T. Large-scale ACDC EMT level system simulations by a real time digital simulator (RTDS) in KEPRI-KEPCO. KEPCO J. Electr. Power Energy 2017, 3, 17-21. [CrossRef]

8. Song, J.; Oh, S.; Lee, J.; Shin, J.; Jang, G. Application of the First Replica Controller in Korean Power Systems. Energies 2020, 13, 3343. [CrossRef]

9. Guo, Y.; Zhang, J.; Hu, Y.; Han, W.; Ou, K.; Jackson, G.; Zhang, Y. Analysis on the effects of STATCOM on CSG based on RTDS. In Proceedings of the IEEE PES ISGT Europe 2013, Lyngby, Denmark, 6-9 October 2013.

10. Lee, H.; Lee, J.; Ko, B.; Hur, K. Internal System Selection for TSAT-RTDS Hybrid Simulation by Electrical Distance. In Proceedings of the 24th International Conference on Electrical Engineering (ICEE 2018), Seoul, Korea, 24-28 June 2018.

11. Zhang, Y.; Gole, A.M.; Wu, W.; Zhang, B.; Sun, H. Development and Analysis of Applicability of a Hybrid Transient Simulation Platform Combining TSA and EMT Elements. IEEE Trans. Power Syst. 2013, 28, 357-366. [CrossRef]

12. Lin, X.; Gole, A.M.; Yu, M. A Wide-Band Multi-Port System Equivalent for Real-Time Digital Power System Simulators. IEEE Trans. Power Syst. 2019, 24, 237-249. [CrossRef]

13. Lin, X. System Equivalent for Real Time Digital Simulator. Ph.D. Thesis, University of Manitoba, Winnipeg, MB, Canada, 2011.

14. Hu, Y.; Wu, W.; Zhang, B.; Guo, Q. Development of an RTDS-TSA hybrid transient simulation platform with frequency dependent network equivalents. In Proceedings of the IEEE PES ISGT Europe 2013, Lyngby, Denmark, 6-9 October 2013.

15. Shu, D.; Xie, X.; Dinavahi, V.; Zhang, C.; Ye, X.; Jiang, Q. Dynamic Phasor Based Interface Model for EMT and Transient Stability Hybrid Simulations. IEEE Trans. Power Syst. 2018, 33, 3930-3939. [CrossRef]

16. Panigrahy, N.; Gopalakrishnan, K.S.; Ilamparithi, T.; Kashinath, M.V. Real-time phasor-EMT hybrid simulation for modern power distribution grids. In Proceedings of the 2016 IEEE International Conference on Power Electronics, Drives and Energy Systems (PEDES), Trivandrum, India, 14-17 December 2016.

17. Palensky, P.; Van Der Meer, A.A.; Lopez, C.D.; Joseph, A.; Pan, K. Co-simulation of intelligent power systems. IEEE Ind. Electron. Mag. 2017, 11, 34-50. [CrossRef]

18. Palensky, P.; Van Der Meer, A.A.; Lopez, C.D.; Joseph, A.; Pan, K. Applied Co-simulation of Intelligent Power Systems: Implementing Hybrid Simulators for Complex Power Systems. IEEE Ind. Electron. Mag. 2017, 11, 6-21. [CrossRef]

(C) 2020 by the authors. Licensee MDPI, Basel, Switzerland. This article is an open access article distributed under the terms and conditions of the Creative Commons Attribution (CC BY) license (http://creativecommons.org/licenses/by/4.0/). 